\title{
ATUAÇÃO DO FARMACÊUTICO NA ATENÇÃO AO PACIENTE IDOSO NA FARMÁCIA COMERCIAL NO MUNICÍPIO DE NOVA IGUAÇU
}

\author{
PHARMACIST'S ROLE IN ELDERLY PATIENT CARE IN THE COMMERCIAL \\ PHARMACY IN THE CITY OF NOVA IGUAÇU
}

\author{
Allan Voigt Siqueira de Abreu ${ }^{1}$ \\ Leonardo Guimarães de Andrade ${ }^{2}$
}

RESUMO: A expectativa de vida vem aumentando com o passar dos anos, com isso, se tem um aumento no número de idosos. A atenção farmacêutica ficou indispensável para além de dispensar o medicamento, melhorar a qualidade de vida do idoso. Este artigo tem como objetivo mostrar a importância da atuação do farmacêutico na atenção ao paciente idoso na farmácia comercial, analisando os principais problemas que o idoso encontra na hora de obter o tratamento, definindo de forma racional e segura, mostrando os principais problemas ao interromper o tratamento. A atenção farmacêutica virou referência na pratica de prevenção a saúde, com ética e seriedade, sempre colocando a qualidade de vida do idoso em primeiro lugar e não na comercialização do medicamento, visando o uso racional e necessário para o tratamento completo. Este estudo mostra que o idoso, por dificuldades no seu dia a dia, acaba demorando adquirir o seu tratamento, em alguns casos, nem iniciando da maneira correta, sendo, portanto essencial, a atenção farmacêutica no acompanhamento, tirando as dúvidas, promovendo saúde e bem estar.

Palavras-chave: Atenção farmacêutica. Cuidados aos idosos. Uso racional.

ABSTRACT: Life expectancy has increased over the years, with this, there is an increase in the number of elderly people. Pharmaceutical care was essential in addition to dispensing the drug, improving the quality of life of the elderly. This article aims to show the importance of the role of the pharmacist in the care of elderly patients in commercial pharmacy, analyzing the main problems that the elderly find it time to obtain treatment, defining rationally and safely, showing the main problems when interrupting treatment. Pharmaceutical care has become a reference in the practice of health prevention, with ethics and seriousness, always putting the quality of life of the elderly in first place and not in the commercialization of the drug, aiming at the rational and necessary use for the treatment complete. This study shows that the elderly, due to difficulties in their daily lives, end up taking a long time to acquire their treatment, in some cases, not even starting in the right way. Therefore, pharmaceutical care is essential in monitoring, clearing up doubts, promoting health and welfare.

\footnotetext{
I Graduando em farmácia pela UNIG-Universidade Iguaçu. E-mail: allanvoigt@gmail.com.

${ }^{2}$ Professor e orientador do curso de Famácia da UNIG-Universidade Iguaçu. Mestre em Ciências do Meio Ambiente Universidade Veiga de Almeida. Mestre em Doenças Parasita Universidad Autônoma de Assuncion. Graduação em Enfermagem e Odontologia. E-mail: Leonard.gui@homail.com.
} 
Keywords: Pharmaceutical care. Elderly care. Rational use.

\section{INTRODUÇÃO}

Por muitos anos o farmacêutico era conhecido como o boticário, realizava diagnósticos, produzia medicamentos e vendia, a grande maioria na época, não possuía nível superior. Com o surgimento da indústria, este profissional foi perdendo seu valor, antes era de grande importância a população, após a indústria, passou a ser visto como um simples balconista (MARQUES, et al., 2017).

Hoje, depois de muitos estudos e dedicação do profissional farmacêutico, contribuindo sempre para o uso adequado e racional dos medicamentos, a atenção farmacêutica ficou indispensável nas farmácias e drogarias, sendo de fácil acesso a população (ARRAYS, 2002, apud FERNANDES; CEMBRANELLI, 2015).

$\mathrm{O}$ ato de atender o paciente, dispensando o medicamento prescrito na receita, vai muito além de entregar o medicamento, existem regras que são analisadas na hora da dispensação pelo profissional. Atenção farmacêutica não se limita apenas em liberar o tratamento, mas em instruir o idoso para facilita-lo na hora de usar, de que maneira usar, e por quanto tempo usar o medicamento, mostrando os benefícios e os possíveis problemas que podem acontecer, caso não siga o tratamento prescrito pelo profissional habilitado (MOURA, et al., 2017).

“O farmacêutico é o profissional responsável por atender as pessoas que necessitam de medicamentos, podendo estes ser ou não prescritos por médicos, dentistas etc., [...]” (MOURA et al.,2017 apud ANGONESI \& PEREIRA, 20II).

“A utilização de medicamentos é considerada uma condição frequente entre os idosos e, ainda que contribua para prolongar e melhorar as condições de vida, pode gerar sérios problemas à saúde, [...]” (STEFANO, et al., 2017).

Alguns idosos possuem certas dificuldades na hora de comprar seus medicamentos, levando assim, o uso excessivo de medicamentos, até mesmo a automedicação. A interação medicamentosa pode ser um problema para o idoso, pelo excesso de medicamentos utilizados na terapia, devendo assim, entender como cada um age no organismo, observando 
a farmacocinética e farmacodinâmica, de modo a prosseguir com o tratamento de forma segura (COUTINHO; SILVA, 2002, apud MARQUES et al., 2017)

A dispensação de medicamentos é o ato profissional farmacêutico, que consiste em proporcionar um ou mais medicamentos, em resposta à apresentação de uma receita elaborada por um profissional autorizado. (BRASIL, 2006, apud SANTOS, 2018)

A atenção farmacêutica ao paciente idoso é fundamental, pois conhecer o seu perfil, é de grande importância na hora de orienta-lo, como usar cada medicamento. "Entre os problemas que costumam ocorrer no uso de medicamentos em idosos, podemos citar os seguintes: escolha inadequada do medicamento, falha ao receber o medicamento, uso inadequado [...]” (PERETTA; CICCIA, 200o).

\section{OBJETIVO GERAL}

Descrever, analisar a atuação do farmacêutico na atenção ao paciente idoso na farmácia comercial de nova Iguaçu.

\section{OBJETIVO ESPECÍFICO}

- Descrever como a atuação do farmacêutico é importante para o idoso em farmácia comercial;

- Analisar os principais problemas que o idoso encontra na hora de obter o seu tratamento;

- Definir qual o tratamento ideal para o uso racional de forma segura;

- Apontar os problemas que poderão ocorrer ao interromper o tratamento.

\section{METODOLOGIA}

Esta revisão descreve a atenção farmacêutica com objetivo de atender ao público idoso, assegurando que façam o tratamento de maneira correta, identificando os problemas que poderá ocorrer. Sendo uma revisão bibliográfica, no período de 2015 a 2019, os estudos foram feitos na base de dados Google acadêmico, SCIELO (Scientific Eletronic Library Online). Esta revisão tem como objetivo relatar como a atuação do farmacêutico é indispensável na farmácia comercial, identificando os problemas e limitações que o paciente idoso tem no seu tratamento, orientando de maneira objetiva para a conclusão do tratamento de forma correta e racional. 


\section{JUSTIFICATIVA}

Este trabalho é para mostrar a importância da atenção farmacêutica na farmácia comercial para o idoso. Muitos idosos apresentam dificuldades na hora de iniciar algum tratamento, seja pela visão, memória, entre outras limitações. Com isso, a atenção farmacêutica é indispensável para atender os idosos, identificando suas limitações, informando os cuidados a serem tomados, orientando a forma correta para fazer o tratamento, de maneira racional e eficaz.

\section{ATENÇÃO FARMACÊUTICA}

O profissional farmacêutico conhecido há vários anos como o boticário, não possuía formação acadêmica, em 1832 iniciou-se o primeiro curso de farmácia no Brasil, especificamente no Rio de janeiro, contudo, ainda por vários anos o profissional farmacêutico continuava a ser conhecido como o boticário. Só em I931, foi estruturada, tendo o seu respeito e reconhecimento, para exercer a profissão farmacêutica, agora sendo somente permitida a pratica farmacêutica, mediante ao diploma reconhecido em instituições oficiais, com a inscrição no conselho de seu estado. (PORTAL FARMÁCIA, 2009).

O farmacêutico por ser um profissional da saúde e de fácil acesso para a população, vem sendo reconhecido e valorizado. Orientando os idosos no uso racional de medicamentos, para evitar certos tipos problemas com os medicamentos, assim promovendo saúde e bem estar, proporcionando vida longa e tranquila ao idoso. (FERNANDES; CEMBRANELLI, 2015)

O uso de medicamentos inadequados pela automedicação, favorece os riscos à saúde. Os dados levantados pelos estudos, indicam que a atenção farmacêutica durante a atuação do profissional, traz melhorias, promovendo a saúde do idoso, com tratamento correto. (RODRIGUES; OLIVEIRA, 2016).

Com isso a atenção farmacêutica virou referência na pratica de prevenção a saúde, de forma ética, visando o compromisso e responsabilidade na recuperação do paciente. É a interação direta do farmacêutico com o usuário, visando uma farmacoterapia racional e a obtenção de resultados definidos e mensuráveis, voltados para a melhoria da qualidade de vida. Esta interação também deve envolver as concepções dos seus sujeitos, respeitada as 
suas especificidades biopsicossociais, sob a ótica da integralidade das ações de saúde (IVAMA et al. 2002:24, apud SANTOS, et al., 2018).

\section{ATENÇÃO AO IDOSO}

O envelhecimento está acontecendo cada vez mais rápido entre a população, com o avanço das tecnologias, a população tende a não fazer exercícios, como atividade físicas, pela facilidade que a internet entrega as informações, entre a vida acelerada e/ou problemas causados pela falta de saúde mental. Apesar de tudo isso, as transformações que ocorrem no organismos do idoso, faz com que, aumente o consumo de medicamento, assim, aumentando também, as possíveis interações medicamentosas. "Escolher o medicamento apropriado para idosos é uma iniciativa importante na prevenção de eventos adversos na geriatria" (SOUZA; SÖHSTEN, 2019).

$\mathrm{O}$ atendimento ao idoso deve ser completo e sempre ativo, o farmacêutico deve sempre compreender todo o conjunto de necessidade para com seu cliente idoso, percebendo que o mesmo necessita de uma atenção dobrada quando ao uso de medicamentos, passando pelo ponto de vista medicamentoso, o mesmo deve se sobressair, entrevistando o paciente para que não reste dúvidas de qual medicamento o mesmo deve ingerir (CONYER, et al., 1996, apud MOURA, et al., 2017).

Contudo, torna-se de grande valor a prova do método terapêutico em tratamento prescrito para o idoso, onde a receita seja clara e especifica nos números de medicamentos, quantas doses diárias, as orientações da dosagem prescrita, isso tudo pode ajudar no tratamento, afim de, evitar erros, algo que é comum de achar em receitas, como as indicações erradas (CARDOSO; PILOTO, 2014).

Facilitar a implantação da atenção farmacêutica ao paciente idoso é uma das
estratégias referenciais aos estabelecimentos farmacêuticos, onde o fator comercial
deve ser substituído pela concepção de serviços avançados de saúde, realizando
ações educativas, no âmbito individual e coletivo, visando à realização da prática
desse serviço como deveria ser, pois qualquer evento indesejável que apresente o
paciente, a farmacoterapia interfere de maneira real ou potencialmente em uma
evolução desejada do paciente" (CARDOSO; PILOTO, 2014).

Todavia, atuação farmacêutico é evitar a automedicação, influenciando o consumo de forma responsável e racional o uso de medicamentos, orientando sobre os possíveis problemas relacionados aos medicamentos, instruindo o idoso a não fazer uso sem a 
recomendação do profissional habilitado a prescrever. Visando sempre o bem estar e saúde do idoso em primeiro lugar, o profissional farmacêutico deve estar voltado ao paciente e não ao que a indústria dita como regra, para obter lucros, atitude que vem sendo frequente em drogarias, onde os medicamentos isentos de prescrição, tem uma crescente com o passar dos anos e estão entre os mais usados pela população (FERNANDES; CEMBRANELLI, 2015).

\section{DISCUSSÃO}

Este artigo relata como a atuação do farmacêutico na atenção ao idoso em drogarias é essencial, mostrando a importância de acabar com as dúvidas, para dar início a um tratamento, sem erros e de modo racional (MOURA, et al., 2017).

Em drogarias, o atendimento torna-se automatizado e, alguns pacientes idosos, com dificuldades na leitura da receita devido a letra do prescritor, ou até mesmo pela visão, acabam não adquirindo todo tratamento solicitado pelo prescritor. (MOURA, et al., 2017).

Alguns idosos, como relatado neste artigo, tem mais facilidade de acesso ao profissional farmacêutico, que dá a atenção necessária que eles precisam, mostrando o porquê se deve adquirir todo o tratamento, para que se tenha o resultado esperado, afim de curar, sem que haja complicações pela falta de algum medicamento e ou interação com outros, pela automedicação (MARQUES, et al., 2017).

Sendo assim, o profissional farmacêutico deve compreender a necessidade de cada idoso, fazendo um pequeno levantamento de sua vida como por exemplo: como se comporta, onde vive, sua qualidade de vida, sua saúde como um todo, avaliando sempre o seu comportamento, para melhor atender as suas necessidades (FERNANDES; CEMBRANELLI, 2015).

\section{CONCLUSÃO}

O número de idosos vem aumentando nesses últimos anos, contudo, o sistema de saúde no município, não consegue atender seus pacientes com toda atenção necessária, deixando-os com dúvidas para iniciar o tratamento, com isso, alguns idosos não seguem o tratamento necessário para melhorar a qualidade de vida, pela falta de atenção na unidade, assim, alguns procuram pela ajuda familiar ou até mesmo optando pela automedicação. Portanto, atuação farmacêutica na drogaria não está relacionada em apenas dispensar um 
medicamento, mas sim, em atender e entender o problema de cada idoso, visando melhorar a sua qualidade de vida, tirando as suas dúvidas em relação a cada medicamento, mostrando utilizar, quais horários tomar e qual a importância de cada um, e se sempre acompanhando se o paciente está concluindo o tratamento indicado.

\section{REFERÊNCIAS}

CARDOSO, D. M.; PILOTO, J. A. R., atenção farmacêutica ao idoso: uma revisão, Brazilian Journal of Surgery and Clinical Research, Vol.9, n.I, pp.6o-66(Dez 2014-Fev 2015). Disponível em: mastereditora.com.br/periodico/2014II30_215818.pdf. Acesso em: 08/o9/2021.

FERNANDES, W. S.; CEMBRANELLI, J. C. Automedicação e o uso irracional de medicamentos: o papel do profissional farmacêutico no combate a essas práticas. Revista Univap - revista.univap.br, São José dos Campos-SP-Brasil, v. 21, n. 37, jul.2015. Disponível em: revista.univap.br/index.php/revistaunivap/article/view/265/259. Acesso em: 2I/o9/2021.

MARQUES, F. E. A; RUFINO, M. D. M; SILVA P. L. C; GOMES, F. M. N; ROLIM, R. F. Assistência farmacêutica: uma reflexão sobre o papel do farmacêutico na saúde do paciente idoso no Brasil, Volume 17, Número 3 ISSN 2447-2131 João Pessoa, 2017 Páginas I29 a I46. Disponível em: temasemsaude.com/wp-content/uploads/2017/10/17309.pdf Acesso em: $17 / 09 / 2021$

MOURA, A. G., et al. A importância da atenção farmacêutica ao idoso, Revista: Ciências da saúde, Faculdade de educação e meio ambiente, V. 8, n.I, 90-98, jan-jun., 2017. Disponível em: pt.scribd.com/document/357370581/artigo-idoso. Acesso em: 17/o9/2021.

PERETTA, M.; CICCIA, G. Reengenharia farmacêutica: guia para implementar a atenção farmacêutica. Brasília: Ethosfarma, 2000. Disponível em: Ceatenf.ufc.br/Artigos/ATENFAR\%20e\%20URM\%20Adriano\%2oMax.pdf. Acesso em I5/o9/2I.

PORTAL FARMÁCIA. Curiosidades: Origem do curso de farmácia e do farmacêutico, jul. 2009. Disponível em: pfarma.com.br/curiosidades/i44-origem-do-curso-defarmacia.html. Acesso em: II/og/2I

RODRIGUES M. C. S.; OLIVEIRA C. Interações medicamentosas e reações adversas a medicamentos em polifarmácia em idoso: uma revisão integrativa. Revista latino-americana de Enfermagem. v. 24, 2016. Disponível em: scielo.br/j/rlae/a/FtSs4nsL4HMBbX8yqgqkkSz/?format=pdf\&lang=pt. Acesso em: 24/09/2I.

SANTOS, J. T. S., A importância da atenção farmacêutica ao paciente idoso: uma revisão, Revista Especialize On-line IPOG, Goiânia - Ano 9, Edição no 15 Vol. or julho/2018. 
Disponível em: ipog.edu.br/wp-content/uploads/2020/II/josiana-tavares-silva-santosaffbehoo2-78I4013.pdf. Acesso em: II/o9/2021

SOUZA, T. S. P.; SÖHSTEN, E. C. F., análise do conhecimento e nível de satisfação do idoso em relação ao farmacêutico e à atenção farmacêutica, Trabalho de Conclusão de Curso apresentado à Faculdade Pernambucana de Saúde-FPS, Pág. 26, Recife, 2019, Disponível em: tcc.fps.edu.br/bitstream/fpsrepo/438/r/TCC\%2oFINAL\%20\%20P\%c3\%93S\%2oBANCA\%202.pdf. Acesso em: 26/o9/2021.

STEFANO, I. C. A., et al., Uso de medicamentos por idosos: análise da prescrição, dispensação e utilização num município de porte médio do estado de São Paulo, Rev. Bras. Geriatr. Gerontol. Rio de Janeiro, 2017; 20(5): 681 - 692, Disponível em: scielo.br/j/rbgg/a/syRQgkLSXddb3cJctKcSzZR/?format=pdf\&lang=pt. Acesso em: Io/o9/2021. 